\title{
Médiévales
}

Langues, Textes, Histoire

70 | printemps 2016

Lieux d'hygiène et lieux d'aisance en terre d'Islam (VII $\mathrm{e}_{-}$ $\mathrm{XV}^{\mathrm{e}}$ siècle)

\section{Aménagements hydrauliques et ornementation architecturale des latrines de Madīnat al-Zahrā' : un indicateur de hiérarchie sociale en contexte palatial}

Hydraulic Development and Architectural Decoration in Madinat al-Zahrä's Latrines : a Social Hierarchy Indicator in Palatial Context

\section{Antonio Vallejo Triano}

Traducteur : Patrice Cressier

\section{OpenEdition}

Journals

Édition électronique

URL : http://journals.openedition.org/medievales/7789

DOI : 10.4000/medievales.7789

ISSN : $1777-5892$

Éditeur

Presses universitaires de Vincennes

Édition imprimée

Date de publication : 23 juin 2016

Pagination : 77-94

ISSN : 0751-2708

Référence électronique

Antonio Vallejo Triano, « Aménagements hydrauliques et ornementation architecturale des latrines de Madīnat al-Zahrā' : un indicateur de hiérarchie sociale en contexte palatial », Médiévales [En ligne],

70 I printemps 2016, mis en ligne le 15 juin 2018, consulté le 23 avril 2019. URL : http://

journals.openedition.org/medievales/7789; DOI : 10.4000/medievales.7789 


\section{Aménagements hydrauliques et ornementation architecturale des latrines de Madīnat al-Zahrā' : un indicateur de hiérarchie sociale en contexte palatial}

Devant la rareté, voire l'absence totale, de témoignages archéologiques rendant compte de l'existence de latrines durant la période wisigothique $^{1}$, on peut se demander si l'apparente introduction de leur usage peu après la conquête arabo-islamique n'est pas un des nombreux aspects de ce qu'il est convenu d'appeler «l'islamisation sociale ».

C'est au VIII ${ }^{\mathrm{e}}$ siècle, en effet, durant l'émirat omeyyade qu'apparaissent les premiers exemples. Il s'agit de latrines incluses dans les installations hygiéniques de la grande mosquée de Cordoue, mais d'autres ont dû exister dans les édifices palatiaux du moment (le qaṣr urbain ou la muniya al-Rușāfa, édifiée par 'Abd al-Raḥmān $\mathrm{I}^{\mathrm{er}}$ ), même si rien n'a été conservé de ces deux sièges du pouvoir. Dans le cas du palais intra-muros (aujourd'hui «Alcázar»), l'unique latrine fouillée a été datée de l'époque califale $^{2}$.

La première latrine documentée par l'archéologie est située dans la mida'a, ou salle d'ablution de la grande mosquée, que fit construire l'émir Hisham Ir $^{\text {er entre }} 788$ et 796. Sa morphologie inclut déjà un orifice rectangulaire ménagé dans une petite plateforme maçonnée, sous laquelle

1. Aucun des établissements wisigothiques importants qui ont été fouillés dans la péninsule Ibérique n'a révélé de latrines : ni Recopolis (L. OLMO éd., Recópolis y la ciudad de la época visigoda, Zona Arqueológica, 9, Alcalá de Henares, 2008), ni le Tolmo de Minateda (S. GuTiÉRrEZ LLORET, «El espacio doméstico altomedieval del Tolmo de Minateda (Hellín, Albacete), entre el ámbito urbano y el rural », Castrum, 6 (Maisons et espaces domestiques dans le monde méditerranéen au Moyen Âge), Rome/Madrid, 2000, p. 151-164), ni Mérida (M. AlbACAlZADO, « Los espacios domésticos en la ciudad visigoda de Emerita (ss. VIVIII) », dans J. Ma ÁlvareZ et P. MATEOS CRUZ éd., Actas del Congreso Internacional 19192010 : El Yacimiento Emeritense, Mérida, 2011, p. 521-546).

2. A. LEÓn MuÑoz et J. F. MURILlo REDONDO, « El complejo civil tardoantiguo de Córdoba y su continuidad en el Alcázar Omeya », Madrider Mitteilungen, 50 (2009), p. 399432 (p. 423, pl. 54b). 
court une conduite d'assainissement ${ }^{3}$. Elle dérive de modèles omeyyades, utilisés encore à l'époque abbasside, dont on connaît de nombreux témoins dans l'architecture palatiale, à Amman ${ }^{4}$, Mshattā, Qașr al-Hayr al-Gharbī, Qașr Kharāna ${ }^{5}$, Qașr al-Hayr al-Sharqī ${ }^{6}$ et, d'une façon générale, dans ce qui a été appelé de manière erronée «châteaux du désert ». Ces latrines étaient conçues pour être utilisées en position accroupie, selon une pratique orientale ; elles diffèrent de celles du monde romain, où l'orifice circulaire et l'élévation de la plateforme dans laquelle il s'ouvrait supposaient un usage assis et dont un exemple dérivé est documenté dans le complexe palatial de Khirbat al-Mafdjar ${ }^{7}$.

On ignore quand s'est effectué le transfert de l'architecture officielle à l'environnement domestique, mais il est sûr qu'il n'est pas antérieur aux dernières années du $\mathrm{IX}^{\mathrm{e}}$ siècle, car on ne retrouve de latrines dans aucun établissement d'époque émirale plus ancien : ni à Shaqunda ${ }^{8}$, ni dans les autres quartiers extra muros de Cordoue mis en place à la même époque 9 . Hors de Cordoue, les premières latrines mises au jour en fouille sont, pour le moment, celles des maisons de Badjdjāna (Pechina, province d'Almería), datées de l'époque califale, « pas plus tard que la première moitié du $\mathrm{X}^{\mathrm{e}}$ siècle ${ }^{10}$.

3. Sur cette fouille, voir P. MARFIL RUIZ, «Avance de resultados del estudio arqueológico de la fachada este del oratorio de 'Abd al-Raḥmān I en la mezquita de Córdoba », Cuadernos de Madīnat al-Zahrā', 4 (1999), p. 175-207 (p. 188).

4. I. ARCE, « The Umayyad hydraulic System at Amman Citadel - Collection, Storage, Distribution, Use and Sewage », dans H. BIENERT et J. HAESSER éd., Men of Dikes and Canals : The Archaeology of Water in the Middle East (Orient-Archäologie Band 13), 2004 ; p. $41-58$ (p. 49,57 , fig. 5c).

5. K. A. C. CReSwell, A Short Account of Early Muslim Architecture. Revised and supplemented by James W. Allan, Tiptree, Essex, 1989, respectivement p. 201-202, 137-138, 99-100.

6. O.Grabar et al.,City in the Desert. Qașral-Hayr East, II.Cambridge, Massachusetts, 1978, photos 24, 28, dessin 13D.

7. K. A. C. Creswell, A Short Account..., p. 199.

8. M. T. CASAL GARCíA, " Características generales del urbanismo cordobés de la primera etapa emiral: el arrabal de Shaqunda », Anejos de Anales de Arqueología Cordobesa, 1 (2008), p. 109-134 (p. 126, 133).

9. Quoiqu'on puisse observer dans certains de ces quartiers hors les murs plusieurs phases constructives émirales, les archéologues insistent sur la difficulté d'identifier des unités d'habitat complètes, du fait de la dégradation et de la fragmentation des structures conservées, et donc sur celle de définir la typologie des différents espaces. Voir par exemple : D. RUíz LARA et al., « La ocupación diacrónica del sector meridional del Yanib al-Garbī de Qurțuba (siglos VIII-XIII). Intervenciones arqueológicas realizadas en el Zoológico Municipal de Córdoba. Análisis de conjunto », Anejos de Anales de Arqueología Cordobesa, 1 (2008), p. 163-200 (p. 172-180).

10. F. CASTILlO GALDEANO et R. MARTíneZ MADRID, « La vivienda hispanomusulmana en Baŷŷāna-Pechina (Almería) », dans La casa hispano-musulmana. Aportaciones de la arqueología, Grenade, 1990, p. 111-128 (p. 115). Dans des villes de nouvelle fondation émirale, comme Jaén ou Murcie, nous n'avons pas d'évidence matérielle de ces éléments ; 
La généralisation de ces infrastructures en contexte domestique est contemporaine de celle de l'adoption quasi systématique de l'organisation spatiale, devenue classique pour les maisons urbaines, en salles barlongues se distribuant autour d'un patio central, accompagnée d'une spécialisation de certains espaces (entrée en double coude, alcôves latérales, latrines, etc. ${ }^{11}$. La latrine acquiert alors pratiquement la morphologie qui sera la sienne jusqu'à la fin de la période nasride. Le fait que, à l'exception de quelques zones, la majeure partie des maisons fouillées - tant dans le centre de la Cordoue califale que dans ses quartiers périphériques de même époque - a comporté des latrines donne une bonne idée de l'extension de l'usage de celles-ci et de l'importance donnée alors aux questions hygiéniques et sanitaires. À partir de ce moment, la latrine se convertit en un élément indispensable de la maison urbaine d'al-Andalus (au contraire du monde rural), et cela quel que soit le programme architectural - reflet du statut social du propriétaire - et le type d'assainissement dans lequel elle s'insère, primaire (le puits perdu) ou plus complexe, à savoir des systèmes d'évacuation planifiés à plus grande échelle ${ }^{12}$.

\section{Les latrines de Madīnat al-Zahrā'}

De toute l'époque califale et même de toute l'histoire d'al-Andalus, les latrines dont la conception fut la plus complexe à tous points de vue sont celles de la zone palatine ( $q a s ̦ r$ ) de Madīnat al-Zahrā'. Là furent introduits et réélaborés les techniques, les formes et les éléments constitutifs de l'architecture d'al-Andalus plus tardive.

Le grand nombre des latrines existant dans l'aire fouillée - trentesix au moins ont été reconnues - surprend d'autant plus qu'elles ne sont

à Jaén, ils n'apparaissent pas dans la phase émirale de Marroquíes Bajos et, à Murcie, les éléments hygiéniques et d'assainissement ne sont pas documentés avec certitude jusqu'au $\mathrm{X}^{\mathrm{e}}$ siècle, même si certains auteurs leur supposent une existence préalable. Pour Jaén, voir : J. L. SerRano PeÑa et alii, « La paleomorfología de Marroquíes Bajos. Primeras propuestas », Arqueología y Territorio medieval, 9 (2002), p. 7-36 (p. 17). Pour Murcie, on pourra confronter les différentes opinions émises sur la chronologie des latrines et des systèmes d'égouts dans I. REKLAITYTE, «El saneamiento en las ciudades andalusíes », Anales de Arqueología Cordobesa, 16 (2005), p. 207-238 (p. 212-214). Elles sont absentes, bien entendu, dans le monde rural.

11. Voir M. Acién Almansa et A. Vallejo Triano, « Urbanismo y Estado islámico : de Corduba a Qurțuba-Madīnat al-Zahrā' " , dans P. CRESSIER et M. GARCÍA-ARENAL éd., Genèse de la ville islamique en al-Andalus et au Maghreb occidental, Madrid, 1998, p. 107136 (p. 128-129) ; ainsi que S. GUTIÉRREZ LlORET, « Gramática de la casa. Perspectivas de análisis arqueológico de los espacios domésticos medievales en la península Ibérica (siglos VII-XIII) », Arqueología de la Arquitectura, 9 (2012), p. 139-164 (p. 148).

12. Le recours à l'un ou à l'autre ne peut se comprendre seulement en termes de niveau de développement urbain, car il existe d'autres contraintes comme la disponibilité en eau, la topographie des lieux, etc. 
pas limitées aux espaces domestiques proprement dits, mais qu'elles sont localisées dans toutes les parties du palais, y compris dans des bâtiments administratifs comme le Salon basilical supérieur $\left(\mathrm{n}^{\circ} 28\right)$, et dans ceux à fonction protocolaire de réception et représentation, comme le Salon de 'Abd al-Raḥmān III (Salón Rico, n 42) et le Pavillon central (n $\left.n^{\circ} 43\right)^{13}$.

La plupart d'entre elles sont situées dans l'un des angles de la bâtisse, de préférence au nord-est, sans qu'il existe de direction unique, d'autres étant disposées au nord, au sud, à l'est ou à l'ouest. Toutes sont fermées par des portes à doubles battants, tout comme les autres pièces. Dans le cas des latrines ouvrant directement sur le patio, la vision frontale est rendue impossible par l'interposition d'une cloison entre la porte et la latrine ellemême, imposant un accès en coude. L'aération et l'éclairage sont facilités par cette ouverture sur le patio ou sur un espace annexe à celui-ci, ainsi que par de petites fenêtres évasées, percées à grande hauteur.

La disposition et la morphologie de la latrine elle-même sont également caractéristiques. Sur l'un des petits côtés de la pièce, le sol est surélevé d'une vingtaine de centimètres et percé d'une fente étroite $(0,10 / 0,18 \mathrm{~m}$ x 0,52/1,00 m). Celle-ci est connectée à l'égout par une petite conduite en pente vers l'intérieur. Dans certains cas, à la base de la plateforme ainsi constituée et dans l'axe de la fente, un trou rectangulaire profond de $16 \mathrm{~cm}$ environ est creusé dans le pavement de la pièce ; il est connecté à l'évacuation de la latrine par un petit orifice vertical percé dans le rebord de la plateforme, et qui adopte souvent la forme d'un arc outrepassé. Il permet d'évacuer l'eau provenant d'une vasque associée au dispositif et destinée à l'hygiène corporelle. Du point de vue décoratif, la plateforme induit une surélévation locale du niveau supérieur du lambris peint en brun-rouge courant autour de la pièce. La plateforme et le trou rectangulaire reçoivent le même pavement que le reste de celle-ci : calcaire violet, marbre ou grès calcaire, selon l'emplacement et la catégorie de l'installation.

Toutes les latrines sont connectées directement au réseau souterrain d'assainissement, où leurs résidus se mêlent aux eaux de pluie et à l'excédent de celles résultant des activités domestiques qui sont récupérées par des écoulements ménagés au centre des patios ${ }^{14}$. En ce sens, il n'existe pas deux systèmes concurrents d'évacuation des eaux, de sorte que les latrines sont fréquemment l'aboutissement des gouttières provenant des toitures, comme en témoignent les saignées pratiquées dans les murs et qui accueillaient

13. On verra le plan de localisation de ces latrines dans A. VALLEJO TRIANO, La ciudad califal de Madīnat al-Zahrā'. Arqueología de su excavación, Cordoue, 2010, fig. 24. Les numéros indiqués sont ceux que portent les différents ensembles monumentaux sur le plan officiel du Conjunto arqueológico de Madīnat al-Zahrā'.

14. Une étude des ramifications de ce réseau d'assainissement du qaṣr et son plan dans A. VAllejo Triano, La ciudad califal..., p. 248-254, fig. 22. 
des tuyauteries formées d'éléments céramiques emboîtés (qui n'ont pas été retrouvés in situ).

\section{Techniques et décoration}

Au-delà des caractères généraux exposés ci-dessus, les latrines du qaṣr répondent à différents niveaux de complexité et de somptuosité, des plus simples - aux matériaux les plus communs - aux plus sophistiquées et luxueuses, présentant un riche mobilier associé. Le principal critère de différenciation est l'existence ou non d'une arrivée d'eau et la possibilité de réguler celle-ci par un robinet.

Sur l'ensemble des latrines identifiées, dix ne comportent aucune infrastructure d'adduction (fig. 1a); pour d'autres, il n'est pas possible de trancher. Mais au moins treize ont effectivement été dotées d'un système d'eau courante servant à l'hygiène et à la toilette ainsi qu'au maintien de la propreté de l'installation (fig. 1b). Ceci conditionne tout à la fois leur morphologie (présence du trou rectangulaire en avant de la plateforme) et l'existence d'un mobilier associé. Comme nous allons le voir, c'est précisément ce qui différencie ces latrines du reste de celles d'al-Andalus, contemporaines et postérieures, car si certaines disposent aussi de petites vasques en pierre ou en céramique, elles ne possèdent pas d'alimentation

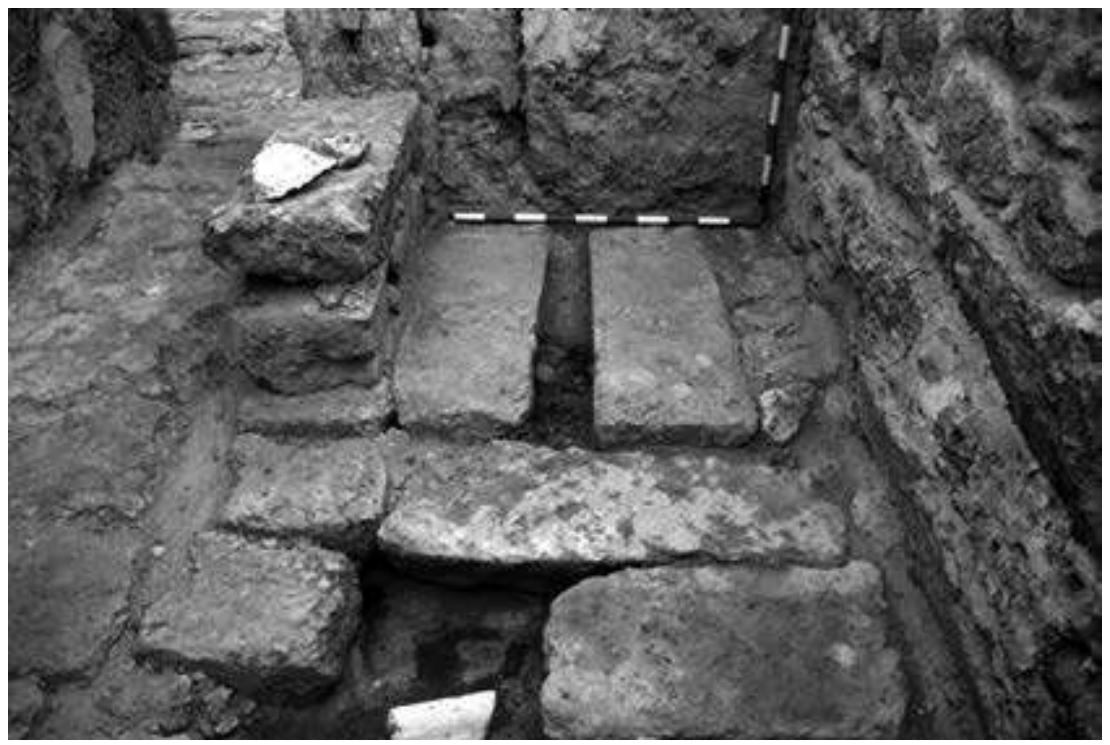

Fig. 1a. Latrine de l'« Espace trapézoïdal » $\left(\mathrm{n}^{\circ} 10\right)$, dans le qaṣr de Madīnat al-Zahrā'

(Cliché S. Escobar Montero) 


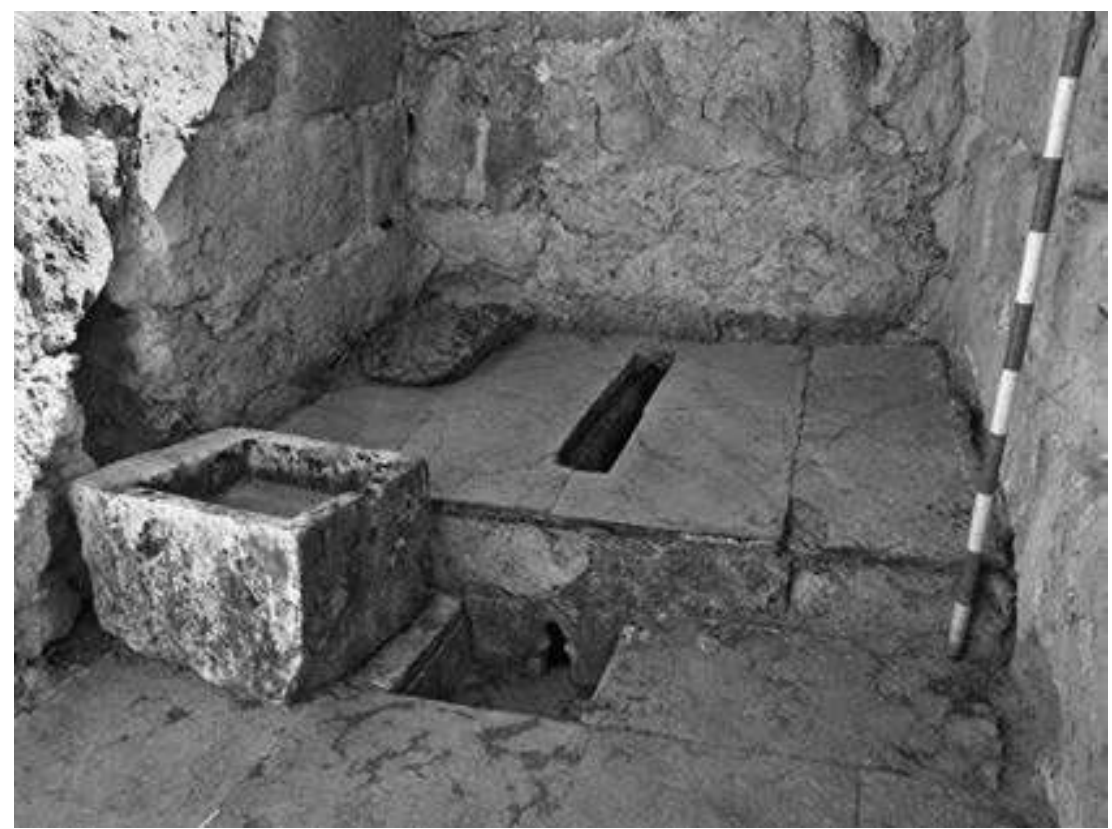

Fig. 1b. Latrine de l' « Édifice supérieur oriental » $\left(\mathrm{n}^{\circ} 7\right)$

(Cliché A. Vallejo Triano)

permanente en eau et doivent être approvisionnées à l'aide de récipients céramiques ${ }^{15}$.

Comment se faisait cette alimentation continue ? La zone du qașr disposait d'un système permanent d'adduction grâce à la remise en fonction d'un aqueduc romain et à son adaptation à de nouveaux besoins ${ }^{16}$. Au sein du palais, la conduite d'eau potable provenant de l'aqueduc desservait les différents édifices jusqu'à la terrasse du jardin supérieur, aboutissement de l'une de ses ramifications. Dans certains cas, comme celui des «Édifices supérieurs » ( $\mathrm{n}^{\circ} 6$ et 7$)$, le point d'arrivée se situait dans l'angle nord-est, au niveau des latrines, et se présente aujourd'hui comme un orifice sans forme définie percé dans le mur, par lequel devait passer la conduite générale

15. Il en est de même pour les latrines nasrides, quoique cela ne soit pas précisé par A. FERnÁNDEZ-PUERTAS, « La casa nazarí en la Alhambra », dans J. NAVARRO PALAZÓn éd., Casas y palacios de al-Andalus. Siglos XII y XIII, Barcelone, 1995, p. 269-286 (p. 274-275). Sur ces latrines avec vasques ou cuvettes, on verra A. VALLEJO TRIANO, La ciudad califal..., p. 256, ainsi que I. REKLAITYTE, Vivir en una ciudad de al-Andalus. Hidráulica, saneamiento y condiciones de vida, Saragosse, 2012, p. 38-41. À Mértola, le récipient destiné à remplir la vasque a été retrouvé associé à celle-ci.

16. Sur l'aqueduc : A. Ventura Villanueva, El abastecimiento de agua a la Córdoba romana. I. El Acueducto de Valdepuentes, Cordoue, 1993 ; sur son réaménagement, certains tronçons gardant leur fonction, d'autres étant transformés en égouts : A. VALLEJO TRIANO, $L a$ ciudad califal..., p. 95-101. 
provenant de l'extérieur (fig. 2a). En ce point, la tuyauterie se divisait en deux circuits : l'un, dirigé vers le centre du patio où était installé un bassin de marbre servant à la consommation humaine et aux activités domestiques, et l'autre, dirigé vers la latrine pour permettre son nettoiement et l'hygiène de l'utilisateur. Dans les deux cas, les tuyauteries de plomb passaient très près sous les sols et les pavements dans lesquels était ménagée une petite tranchée.

C'est la disposition qui prévaut dans la majorité des groupes d'habitations du qașr. Mais, dans les plus prestigieux d'entre eux - et seulement là -, le point d'entrée de la conduite d'adduction est une niche

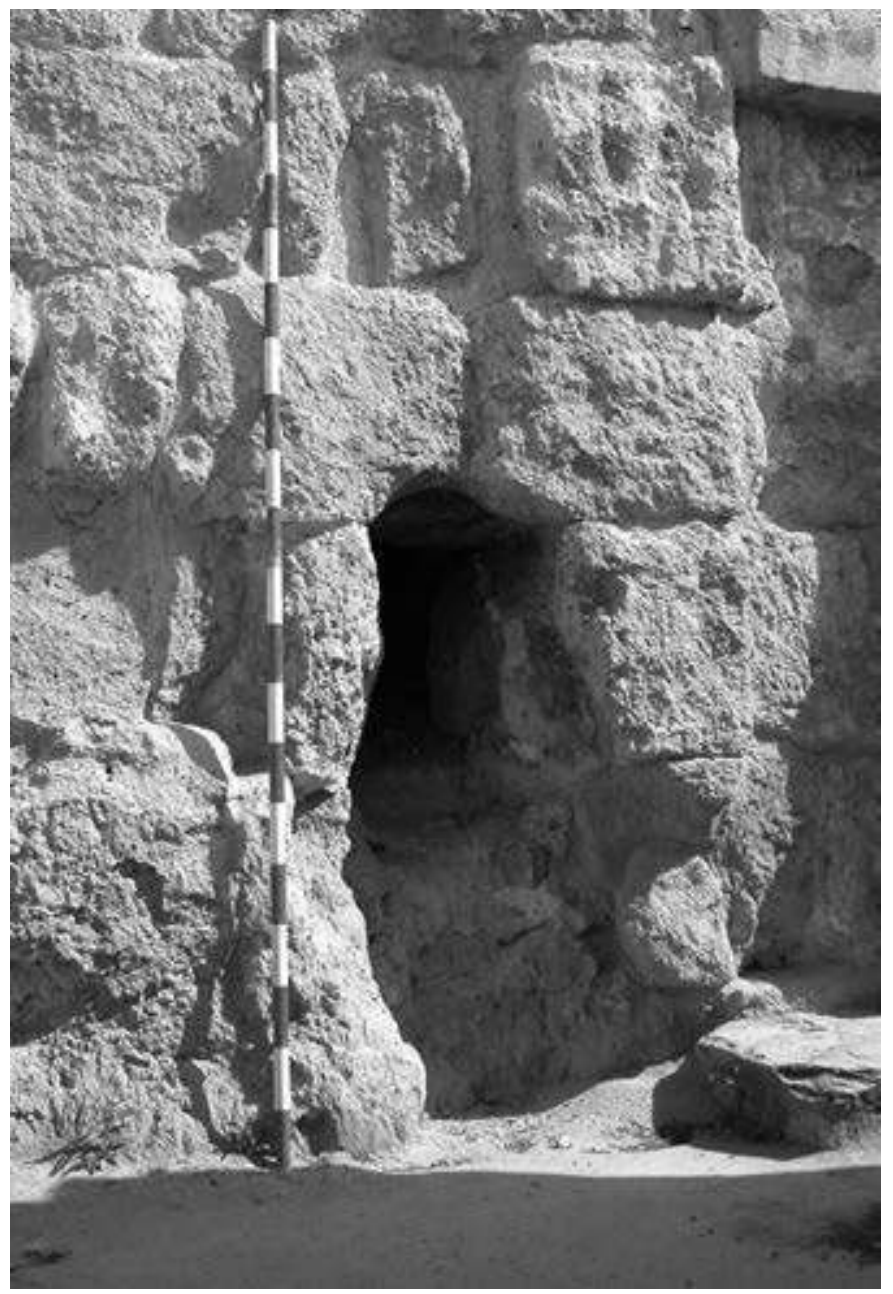

Fig. 2a. Entrée de l'adduction d'eau dans l'« Édifice supérieur oriental » $\left(\mathrm{n}^{\mathrm{o}} 7\right)$ (Cliché S. Escobar Montero) 
rectangulaire, parfaitement aménagée dans l'appareil de pierre de taille et située dans le patio - comme à la Maison de Dja 'far $\left(\mathrm{n}^{\circ} 13\right)$, résidence du hadjib (chambellan) de l'État omeyyade entre 961 et 971 - ou dans la latrine - comme dans les pièces annexes au Salon de 'Abd al-Raḥmān III ( $\left.n^{\circ} 46\right)$, d'usage califal - (fig. 2b). Ces niches sont placées à un peu plus d'un mètre au-dessus du sol et il y confluait généralement plusieurs tuyauteries depuis la base des murs, ainsi qu'en témoignent les saignées pratiquées pour les encastrer.

La somptuosité qu'on observe dans tous les composants du système hydraulique de Madīnat al-Zahrā' est également manifeste dans les latrines utilisées par le calife, où les niches sont pourvues d'un encadrement richement décoré, à la mesure du statut de ses utilisateurs. Il s'agit d'arcs ornementaux en marbre, dont les fouilles ont fourni à ce jour au moins dix exemplaires, auxquels s'ajoute un onzième d'interprétation douteuse, et au

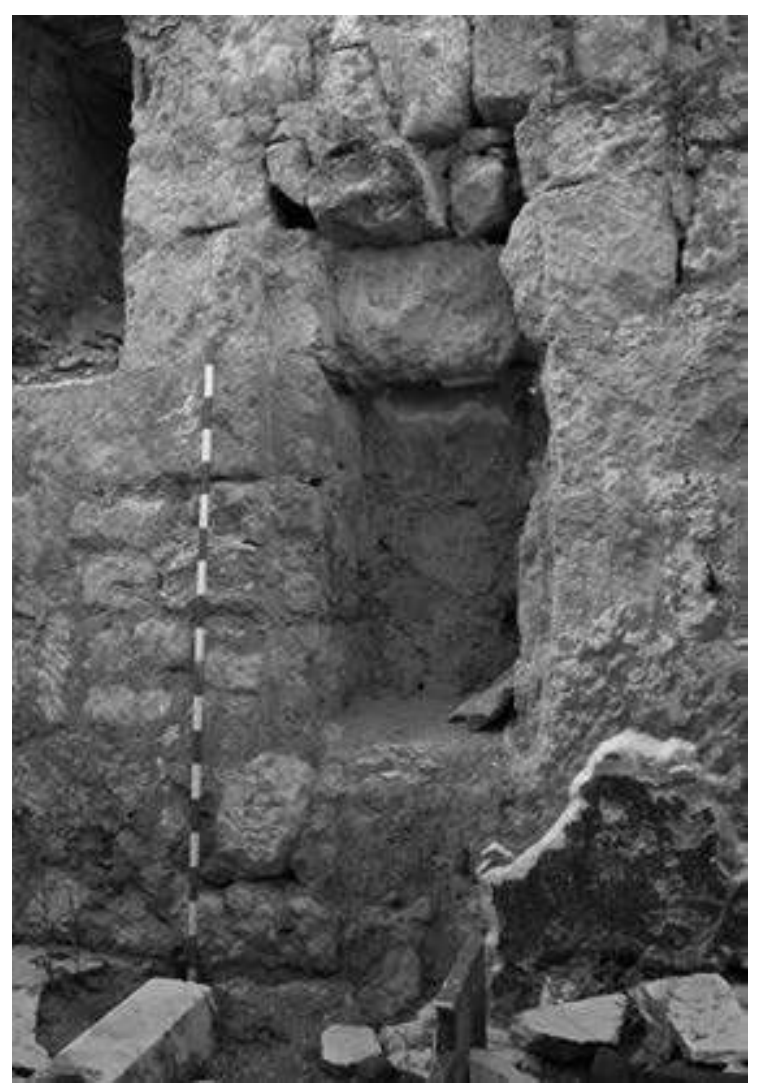

Fig. $2 \mathrm{~b}$. Niche par où pénétrait l'adduction d'eau dans une latrine des pièces annexes au Salon de 'Abd al-Raḥmān III ( ${ }^{\circ}$ 46)

(Cliché M. Pijuán Martínez) 
sein desquels se distinguent deux séries typologiques ${ }^{17}$. Ils constituent un des éléments les plus singuliers de la décoration architecturale califale et, si l'on ne connaît pour l'instant que ceux de Madīnat al-Zahrā', on ne peut écarter qu'ils aient été présents aussi dans d'autres complexes palatiaux comme l'Alcázar de Cordoue ${ }^{18}$. Tous sont élaborés en une seule pièce, dans des plaques de marbre de moins de $10 \mathrm{~cm}$ d'épaisseur, et ils présentent habituellement un fin décor sculpté sur tous les éléments constitutifs de l'arc (archivoltes, écoinçons, encadrement, parfois tympan) ou ses supports (colonnettes). Ils sont souvent décorés d'inscriptions commémoratives à la louange du calife. Toute la composition est enfermée dans un double bandeau. L'inscription peut courir sur l'encadrement, parfois sur le linteau supportant le tympan, voire même sur les tailloirs.

Certains de ces arcs sont complètement perforés, tels les trois exemplaires datés de 961 que nous identifions comme ayant appartenu au bain annexe à la « Maison du Bassin » $\left(\mathrm{n}^{\circ} 14\right)^{19}$. Ils devaient intervenir dans une composition tripartite et orner les trois ouvertures au travers desquelles passait la vapeur d'eau de la chaudière à la salle chaude.

Le tympan d'autres arcs de ce type est en revanche massif. On en conserve quatre exemplaires pour l'ensemble des palais, dont trois proviennent des pièces annexes au Salón Rico $\left(\mathrm{n}^{\circ} 46\right)$, espace exclusivement califal. Le travail de reconstruction qui a été effectué sur ces trois arcs permet d'affirmer que deux d'entre eux étaient employés dans les latrines de ces pièces, où les deux niches qu'ils ornaient sont d'ailleurs encore visibles ${ }^{20}$. Le petit arc avec tympan décoré de rinceaux et d'une petite coquille centrale, et porteur d'une inscription au nom de 'Abd al-Raḥmān III doit correspondre, quant à lui, à la latrine contiguë à la nef orientale du Salón Rico ${ }^{21}$, tandis qu'un autre, anépigraphe et dont le tympan porte une double

17. A. Vallejo Triano, La ciudad califal..., p. 243-244.

18. C'est peut-être au siège du pouvoir que furent ces palais qu'appartenait le petit arc complet conservé dans le cloître de la cathédrale de Tarragone, dont il a été dit qu'il s'agissait d'une pièce cordouane rapportée en butin et réemployée. Il doit avoir été sculpté à Madīnat al-Zahrā'. Son inscription le date de 960-961 et précise qu'il fut réalisé sur ordre du calife 'Abd al-Raḥmān III. Voir M. OCAÑA JiMÉNEZ, El cúfico hispano y su evolución, Madrid, 1970, p. 34.

19. La «Vivienda de la Alberca » $\left(n^{\circ} 14\right)$. A. VAllejo TRIANO, « El baño próximo al Salón de 'Abd al-Raḥmān III », Cuadernos de Madīnat al-Zahrā', 1 (1987), p. 141-165 (p. 145-147).

20. L'hypothèse selon laquelle ces arcs s'intégraient aux niches de ces pièces annexes avait déjà été émise par F. HERNÁNDEZ, Madinat al-Zahra. Arquitectura y decoración, Grenade, 1985, p. 52.

21. La largeur de l'arc est de 0,92 $\mathrm{m}$ et le mur avait été recreusé de $0,94 \mathrm{~m}$ pour le fixer. La lecture de l'inscription a été faite par M ${ }^{\mathrm{a}} \mathrm{A}$. MARTíNEZ NÚÑEZ, « La epigrafía del Salón de 'Abd al-Raḥmān III », dans A. VALLEJo (coord.), Madīnat al-Zahrā'. El Salón de 'Abd al-Rahmmān III, Cordoue, 1995, p. 109-152 (p. 128-129, n 30). 
coquille, devait être employé dans la latrine située au nord du « Patio du Bassin ${ }^{22}$, fonctionnellement associé au bain (fig. 3a).

Que pouvaient bien recevoir, alors, les niches pourvues de ces petits arcs décoratifs? Nous n'avons pas retrouvé d'antécédents convaincants de ces éléments dans le monde omeyyade oriental ni abbasside ; il en existe, en revanche, plus tard à l'époque nasride et plus concrètement à l'Alhambra de Grenade, où ils sont associés à l'approvisionnement en eau potable. On les trouve aux jambages des accès aux salles principales du palais de Comares et à celui de la Cour des Lions. Les niches s'ouvrent par des arcs similaires en marbre ou en stuc. Les inscriptions qu'elles portent indiquent qu'elles abritaient des cruches (fig. 3b) ${ }^{23}$.

En outre, des arcs semblables ont été disposés en avant des jets d'eau alimentant les cuves d'eau chaude du bain du palais de Comares. À Madīnat al-Zahrā', les renfoncements associés ne paraissent avoir enfermé ni récipient, ni vasque ni jet d'eau, mais plus simplement le mécanisme régulateur, c'est-à-dire les robinets d'entrée et de sortie de l'eau vers la latrine et vers des espaces annexes de l'édifice ${ }^{24}$.

La présence de ces arcs dans les latrines d'utilisation califale vient compléter le riche programme architectural et ornemental mis en place dans ces espaces particuliers : il faudrait y inclure les éléments figuratifs en bronze qu'étaient les robinets eux-mêmes. Ces pièces sont les seules qui n'ont pas été retrouvées en fouille, mais il en est toujours ainsi du mobilier métallique somptuaire, qui fait systématiquement l'objet de récupération. Ils n'étaient sans doute pas très différents des robinets à valve rotative du monde fatimide contemporain ${ }^{25}$.

Comment l'eau parvenait-elle jusqu'à ces installations ? Dans les latrines alimentées en eau courante du qașr, nous avons vu qu'elle circulait par des conduites de plomb prises dans la maçonnerie des murs, du côté gauche en entrant dans ces pièces, au-dessus de la plateforme, comme on peut le constater à partir des saignées faites dans les murs. En avant de cette plateforme, l'eau venait remplir une vasque. Deux de ces vasques, en pierre calcaire, ont été retrouvées en place lors de fouilles anciennes (fig. 1b), tout comme des vestiges de la base maçonnée supportant d'autres exemplaires, également en place, et un autre bassin de ce type, en marbre cette fois,

22. «Patio de la Pila ». Cette pièce est quasi complète : hauteur $=1,215 \mathrm{~m}$, largeur $=$ $0,81 \mathrm{~m}$, le mur ayant été recreusé de $0,85 \mathrm{~m}$ pour le recevoir.

23. D. CABAnelas y A. Fernández Puertas, « Los poemas de las tacas del arco de acceso a la Sala de la Barca », Cuadernos de la Alhambra, 19-20 (1984), p. 61-149 (p. 148).

24. Pour nous, il ne fait pas de doute que la fontaine de l'espace nommé aujourd'hui « Patio de la Pila » voyait réguler son fonctionnement à partir du robinet situé dans la latrine située au nord, car la tuyauterie l'alimentant provient de cette pièce ainsi que le montrent les empreintes de son tracé.

25. Voir par exemple Trésors fatimides du Caire, Paris, 1998, nº 47, p. 120. 
Fig. 3a. Arc décoratif de la latrine des pièces annexes au Salon de 'Abd al-Raḥmān III (n' 46) (Cliché M. Pijuán Martínez)

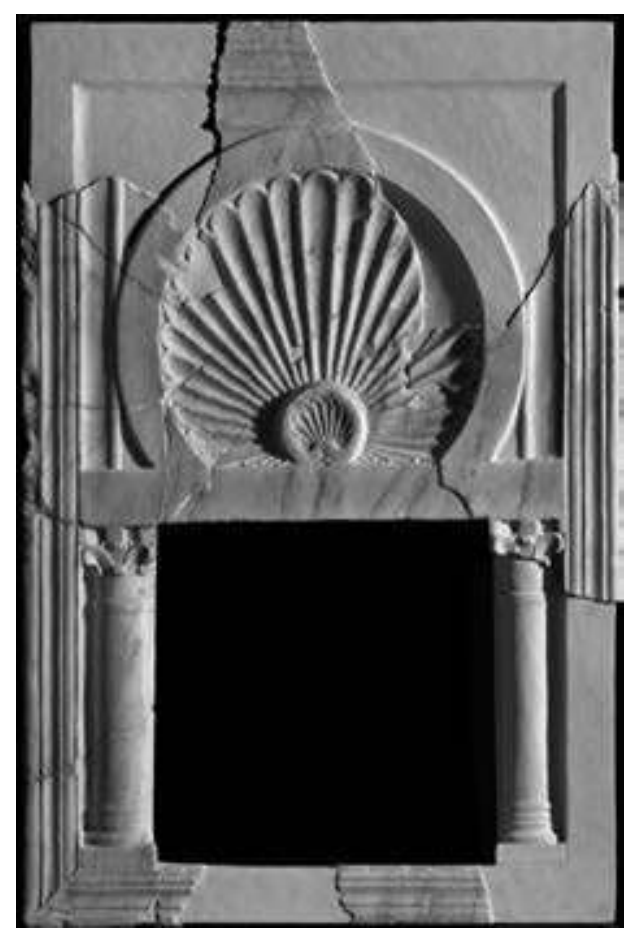

Fig. 3b. Arc décoratif dans le palais nasride dit « de Comares »

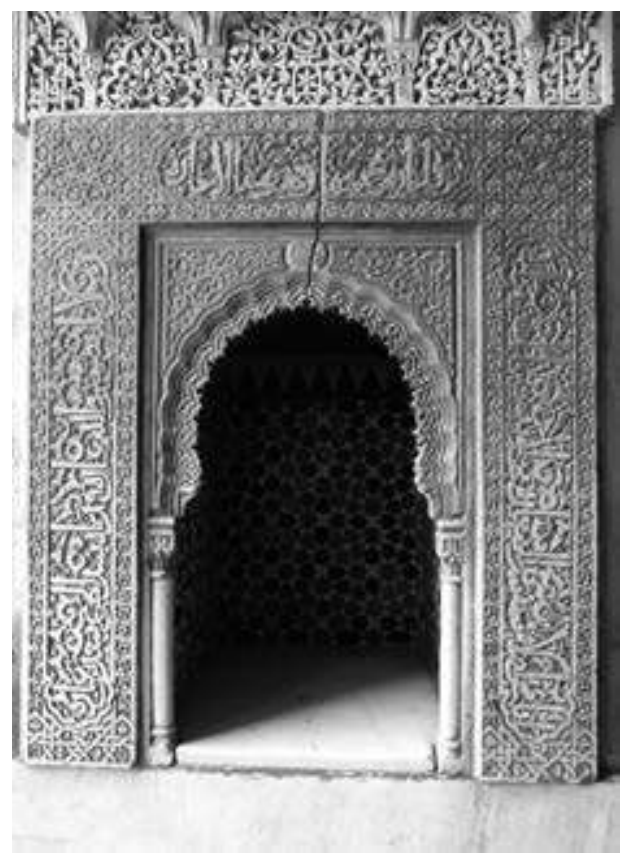

(Alhambra de Grenade)

(Cliché A. Vallejo Triano) 
complet mais hors contexte ${ }^{26}$. À cela, il faut ajouter divers fragments de vasques de marbre, recueillis lors de fouilles plus récentes et des supports partiellement conservés dans les latrines elles-mêmes ou à proximité, et dont la reconstruction a permis de mieux connaître le fonctionnement du système hydraulique de ces espaces et la fonction des différents éléments.

Ces bassins de marbre, de forme carrée ou rectangulaire et de plus grande taille que les précédents, appartenaient aux latrines des édifices les plus prestigieux du palais ${ }^{27}$. Leur morphologie est caractéristique (fig. $4 \mathrm{a}$ et b) : ce sont des objets lisses, de section recticurviligne, parois divergentes et légèrement campaniformes. Leur base-piédestal prismatique est de hauteur variable et présente un jeu de moulures à sa jointure avec les parois. Le bord supérieur de celles-ci, épaissi, fait angle droit. Sa mouluration se compose de deux scoties enserrant un tore disposé au sommet. Sur l'un des côtés (court ou long), une entaille sert de verseur. Toutes les surfaces sauf la base sont polies.

Il faut associer à ces bassins d'autres pièces de marbre que nous identifions comme supports des jets d'eau les alimentant. Deux types se distinguent. L'un d'eux est un bloc de marbre prismatique, partiellement creusé pour recevoir une tuyauterie et travaillé sur l'une de ses faces pour s'adapter à la forme de la vasque, ce qui confirme qu'il faisait bien partie du système d'adduction. Ces pièces sont semblables à celles qui contribuaient à l'alimentation en eau des cuves circulaires retrouvées en divers lieux du palais $^{28}$. Le second type de support est caractéristique des deux latrines califales des pièces annexes au Salón Rico $\left(\mathrm{n}^{\circ} 46\right)$. Dans chacune d'elles est conservé un élément de marbre en tronc de pyramide allongé, de faible hauteur (fig. 4a1), qui servait de couvercle à un caisson délimité latéralement par deux plaques de marbre à l'intérieur duquel était disposée la tuyauterie de plomb (fig. 4a2). Celle-ci émergeait de la pièce en tronc de pyramide par un orifice situé à l'extrémité de celui-ci, là où devait être installé le jet proprement dit $^{29}$. En ce point, ces objets ont été rabaissés pour s'adapter à la morphologie de ce type de vasque au niveau desquelles ils venaient s'accoler. À l'extrémité opposée, une de ces pièces en tronc de pyramide conserve les traces d'enduit rouge qui marquent le niveau auquel elle était

26. R. CASTEJón y MARTíneZ de ARIZALA, Excavaciones del Plan Nacional en Medina Azahara (Córdoba). Campaña de 1943, Madrid, 1945, p. 22 et 42, pl. IV.

27. Des deux plus complètes, l'une devait être employée dans les pièces annexes au Salon de 'Abd al-Rahmān III (longueur $=0,77 \mathrm{~m}$, largeur $=0,64 \mathrm{~m}$, hauteur $=0,23 \mathrm{~m}$ ) et l'autre au «Patio de los Pilares » (Patio des Piliers, $\mathrm{n}^{\circ}$ 16) (1,17 m x 0,83 m x 0,39 m).

28. Par exemple dans le Patio du Bassin - dans les pièces annexes au Salon de 'Abd al-Raḥmān III ( ${ }^{\circ}$ 46) - et dans la maison de Dja ‘far (nº13).

29. Les deux pièces conservées présentent des dimensions différentes. Celle de la latrine accolée au Salon mesure $0,36 \mathrm{~m}$ de large à la base et $0,195 \mathrm{~m}$ à la surface supérieure, $0,985 \mathrm{~m}$ de hauteur et $0,355 \mathrm{~m}$ de long. Celle de la latrine située au nord du Patio du Bassin, respectivement $0,295 \mathrm{~m}, 0,18 \mathrm{~m}, 0,14 \mathrm{~m}$ et $0,70 \mathrm{~m}$. 
encastrée dans le mur. Il ne fait pas de doute que ces éléments ont la même fonction de support de verseur que les blocs de marbre cubiques décrits plus haut.

Quoiqu'aucune des deux latrines dont il est ici question n'ait conservé tous ces éléments, le faisceau d'indices recueillis et analysés permet d'assurer avec une bonne certitude le fonctionnement de leur système d'adduction et de nettoyage (fig. 5). L'eau était conduite par une tuyauterie de plomb jusqu'à la droite de l'usager, où était situé le robinet régulant son débit. Elle pénétrait dans le support de marbre (cubique ou en tronc de pyramide), d'où elle jaillissait dans la vasque qui y était accolée.
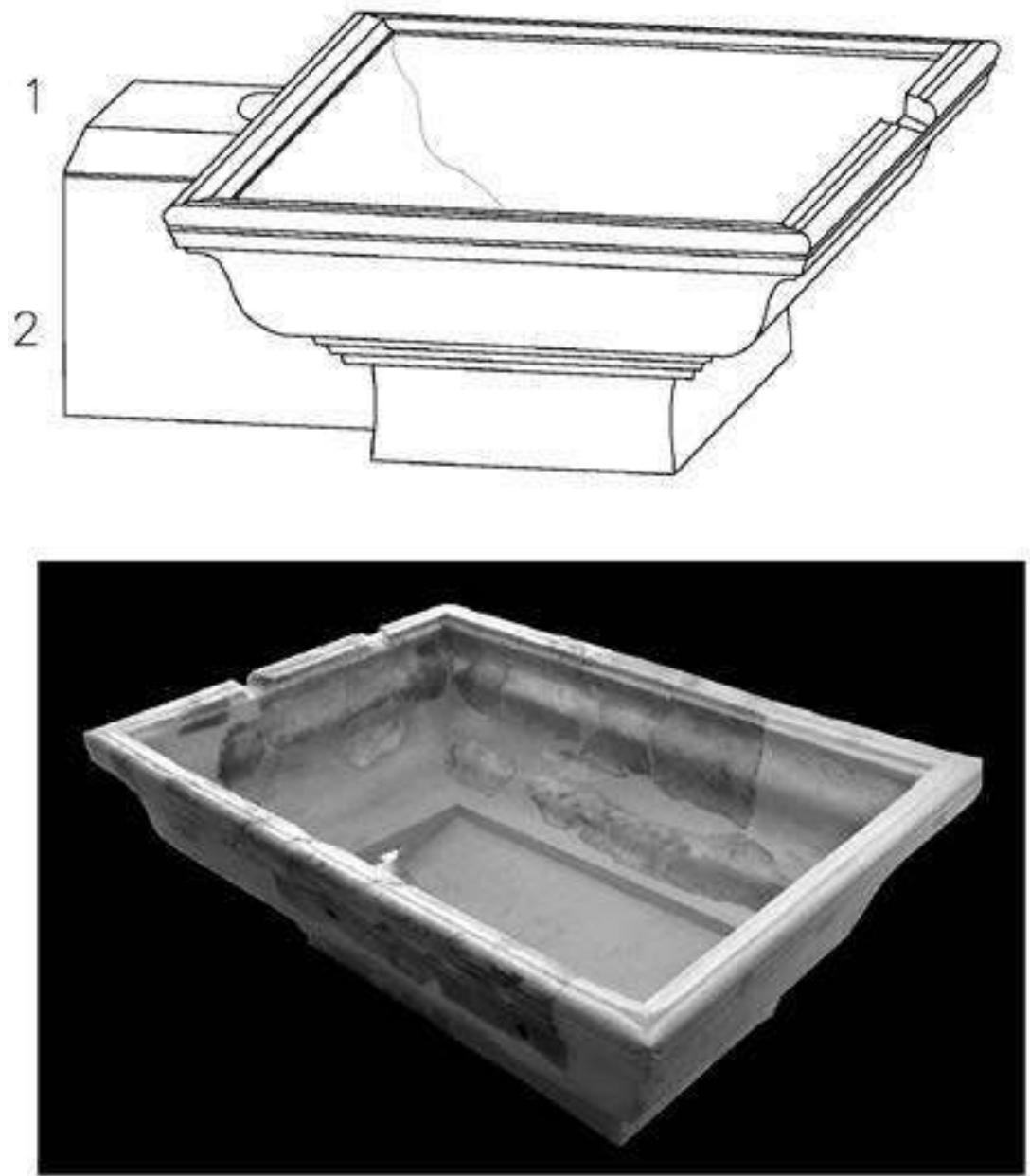

Fig. 4 ab. Vasque et support de robinet d'une latrine califale $\mathrm{du} \ll$ Patio des Piliers $»\left(\mathrm{n}^{\circ} 16\right)$

(Cliché M. Pijuán Martínez 


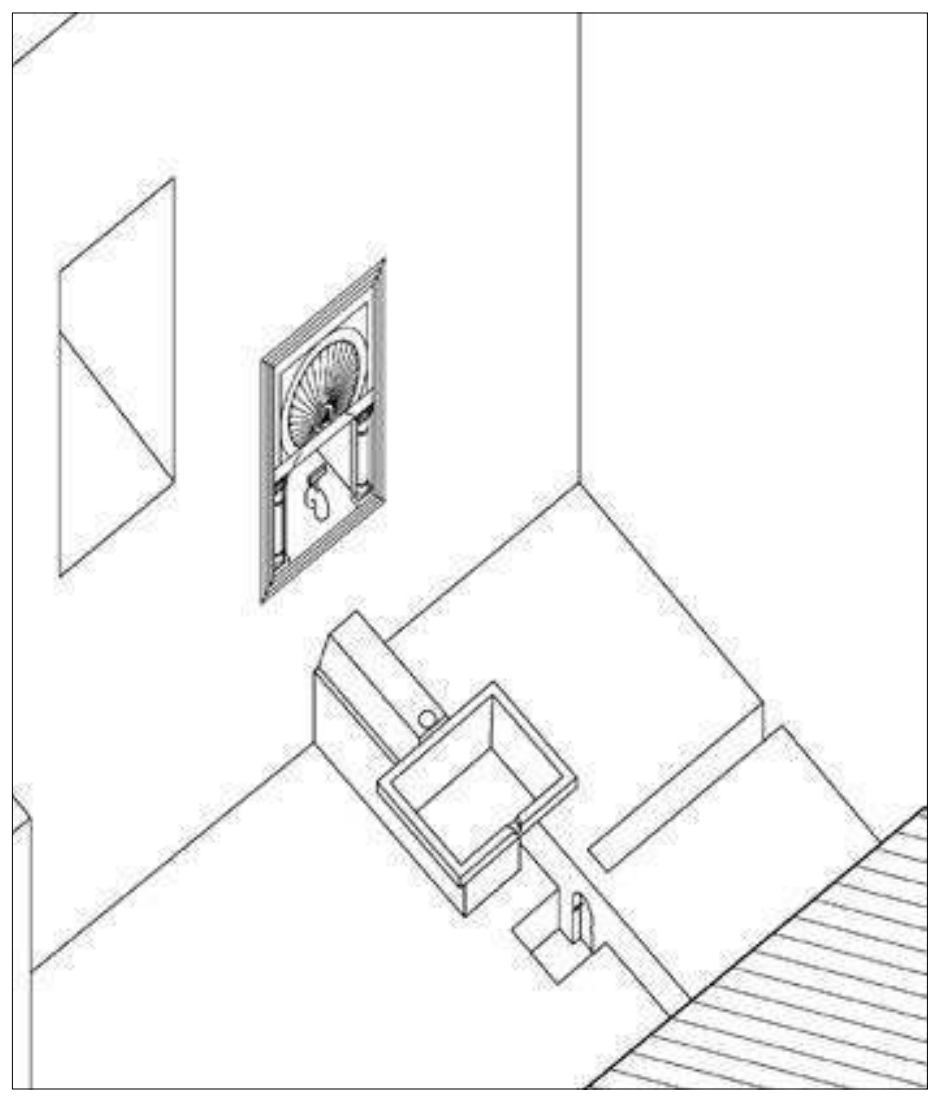

Fig. 5. Schéma de fonctionnement d'une latrine califale

Ces vasques se vidaient à leur tour dans le défoncement quadrangulaire du sol situé en avant de la fente de la latrine et à la base de laquelle elle disparaissait dans le conduit d'évacuation, garantissant ainsi la propreté de l'installation. Contrairement aux autres latrines de Madīnat al-Zahrā', associées à des édifications importantes, c'est l'usager de ces latrines d'utilisation califale qui pouvait contrôler le débit de l'eau en manœuvrant le robinet placé dans les niches pourvues d'arcs ornementaux.

La finalité de ces installations était donc double : d'une part, pourvoir aux besoins de l'usager, tant dans l'accomplissement de sa toilette que de son hygiène intime et, d'autre part, permettre le nettoyage de l'ensemble de cette installation. En ce qui concerne cette pratique, il faut remarquer que les vasques étaient toujours situées à la droite de l'utilisateur, ce qui pourrait l'avoir obligé à utiliser sa main droite pour effectuer sa toilette intime, à l'encontre donc des préceptes de pureté exprimés dans le hadith bien connu, recueilli par le célèbre traditionaliste al-Bukharī dans la première moitié du 
$\mathrm{IX}^{\mathrm{e}}$ siècle, qui défend d'utiliser cette main pour cette fonction ${ }^{30}$. Ceci amène à penser que l'utilisation des vasques ne se faisait pas dans le sens de celui de la latrine elle-même, mais perpendiculairement, à la manière d'un bidet moderne. La hauteur à laquelle étaient placés ces bassins confirmerait cette hypothèse et s'accorde à la fonction que nous leur attribuons ${ }^{31}$.

La présence de l'eau courante révèle le haut degré de sophistication du système car, si l'on en juge par les autres exemples de latrines qui ont été conservées en al-Andalus, ce recours n'est plus documenté après le califat, ni même en contexte palatial, et il n'est plus limité qu'aux infrastructures hygiéniques des mida'a-s des mosquées ${ }^{32}$. Certaines de ces latrines plus récentes sont pourvues de petites vasques en pierre ou en céramique, mais n'ont pas d'alimentation permanente et celles-ci doivent être remplies avec un autre récipient, adapté au transport de l'eau. Même à l'Alhambra de Grenade, presque toutes les latrines possèdent une petite niche, à la hauteur du sol et le plus souvent à la droite de l'utilisateur, où devaient prendre place la cuvette et la cruche. C'est le cas tant dans les maisons modestes de la qașaba que dans celles du vieux Mashwar d'Ismā'îl I Ir (1314-1325), ou dans celles, plus récentes, des palais de Comares et de la cour des Lions, datés de la seconde moitié du XIV ${ }^{\mathrm{e}}$ siècle. Aucune des latrines de l'Alhambra ne s'écarte de ce modèle car aucune ne dispose d'eau courante ${ }^{33}$.

30. AL-BUKHARI, Les Traditions islamiques, traduites de l'arabe avec notes et index par O. HoudAS et W. MARÇAIS, t. I, Paris, 1984 (rééd.), p. 71-72.

31. La hauteur de l'un d'eux et de son piédestal incorporé est de $0,39 \mathrm{~m}$, similaire à celle des actuels bidets. Par cette comparaison, nous souhaitons montrer comment la fonction peut déterminer la morphologie et les dimensions d'objets culturellement distincts mais conçus pour un même usage.

32. Dans ces installations, l'eau destinée à la toilette était située dans des vasques de maçonnerie, comme à Cordoue (A. MONTEJO CÓRDOBA, «El pabellón de abluciones oriental de la Mezquita Aljama de Córdoba correspondiente a la ampliación de Almanzor », Cuadernos de Madīnat al-Zahrā', 4 (1999), p. 209-231), ou dans une fontaine au centre de chacun des corps de latrines, comme dans la mida'a (salle d'ablution collective associée à la mosquée) almohade de Séville (M. VERA ReINA, «La mida' », dans Sevilla Almohade, Séville/Rabat, 1999, p. 107-109). L'utilisateur devait alors prélever l'eau avec un récipient et s'isoler ensuite dans la latrine pour procéder à sa toilette.

33. Dans ces deux palais, A. Fernández-Puertas a proposé de distinguer les espaces d'habitation de ceux de représentation par la présence de latrines dans ces derniers : A. FERNÁNDEZ-PUERTAS, « La Alhambra. El Alcázar del Sultán (hoy Comares) y el Alcázar del Jardín Feliz (hoy Leones), según los Diwanes de Ibn al-Jațīb e Ibn Zamrak », dans Ibn Jaldún. El Mediterráneo en el siglo XIV : Auge y declive de los Imperios. La Península Ibérica. El entorno mediterráneo, Grenade, 2006, p. 101-127 (p. 107, 117). 


\section{La latrine, indicateur de hiérarchisation sociale}

Comme nous pensons l'avoir démontré, à Madīnat al-Zahrā', la présence de latrines permet de caractériser clairement les espaces et les individus ou les groupes qui les occupent. D'une part, elles signalent la catégorie et le statut social de l'usager en fonction de leur localisation dans le contexte bâti - associée ou non à une pièce de repos -, de leur superficie et de la qualité de leur décor et de ses matériaux. D'autre part, elles mettent en évidence, mieux que tout autre pièce d'un bâtiment, l'organisation et la hiérarchisation de celui-ci, contribuant ainsi à définir certaines des habitations palatiales non comme des résidences familiales - ce que suggère pourtant leur typologie -, mais comme des espaces où se déroule une activité domestique dirigée par un fonctionnaire, qui vit dans cet espace, et réalisée par des opérateurs qui n'y résident pas.

La multiplication de ces pièces dans un même édifice - souvent deux par complexe, comme nous l'avons signalé - peut impliquer, dans certains cas, la présence d'un grand nombre d'utilisateurs, mais, en d'autres cas, cela indique l'existence d'espaces aux fonctions différenciées, de résidence et de travail, occupés ou desservis par un personnel obéissant à une stricte hiérarchie. C'est dans cette perspective que doit être interprétée une bonne partie des latrines du palais (fig. 6). Certaines servent à l'ensemble du bâtiment et s'ouvrent directement sur un patio central, une petite cour annexe ou un espace intermédiaire, ce qui signifie qu'elles sont d'utilisation partagée par tous les résidents ou usagers, quelle que soit leur catégorie. D'autres sont associées étroitement aux pièces considérées comme de repos, même lorsqu'elles ne sont pas mitoyennes ${ }^{34}$.

Les différences entre les deux types de latrines, coexistant parfois au sein d'un même édifice, rendent compte d'une différence de catégorie sociale de leurs usagers respectifs. La « Maison orientale » $\left(\mathrm{n}^{\mathrm{o}} 12\right)$ [fig. 6], par exemple, possède deux latrines. L'une d'elles est fréquentée par le personnel de service qui travaille dans ce bâtiment. Elle est double et ne dispose pas d'eau courante, ne pouvant compter que sur les gouttières évacuant l'eau de pluie des toitures pour un nettoyage éventuel des conduites, mais évidemment pas pour l'hygiène de l'utilisateur. En plus de cette latrine double, il en existe une autre, associée à l'espace de vie du fonctionnaire qui contrôlait les activités menées dans cette maison (en l'occurrence celle d'une cuisine collective). Cette seconde latrine diffère beaucoup de la première : elle est pavée de marbre et, surtout, elle est dotée d'un dispositif d'eau courante, ce qui implique la présence d'une vasque du même matériau pour l'hygiène intime du personnage. Cette situation se retrouve dans la maison mitoyenne (fig. 6), qui compte deux latrines,

34. A. Vallejo Triano, La ciudad califal..., p. 255. 


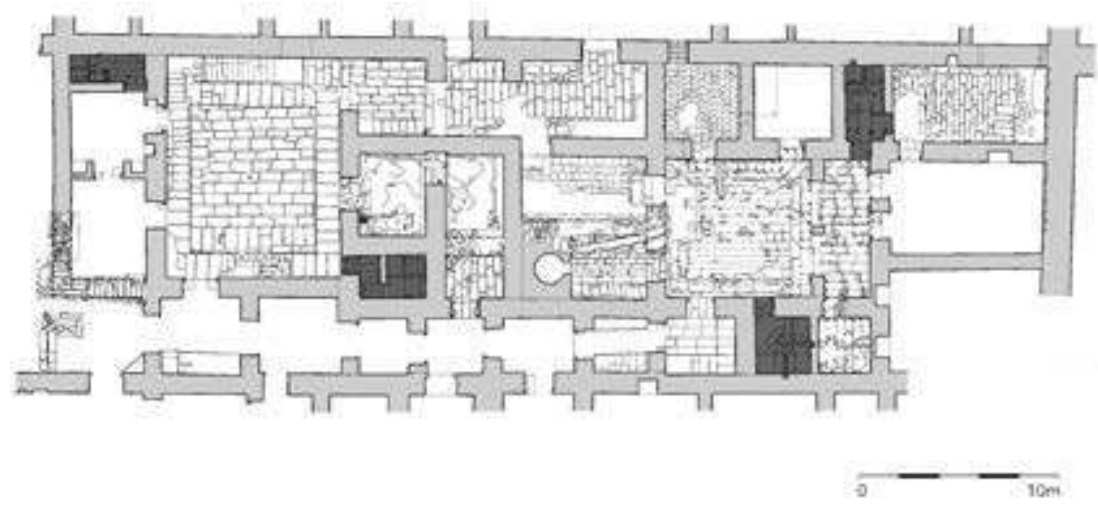

Fig. 6. Plan des habitations de service et implantation des latrines « Maison occidentale» $\left(\mathrm{n}^{\circ} 11\right)$, à gauche, et « Maison orientale » $\left(\mathrm{n}^{\circ} 12\right)$, à droite.

une de chacun des types qui viennent d'être décrits. Nous sommes alors en mesure d'affirmer qu'à Madīnat al-Zahrā' la latrine indique mieux que la pièce d'habitation le statut et la hiérarchie des habitants du palais. À ces deux types s'ajoute celui d'usage exclusivement du calife, le seul doté de robinet permettant le contrôle du débit de l'eau.

En somme, le système hygiénique du palais répond à trois situations distinctes. D'un côté, des latrines sont utilisées par le personnel de service ; elles ne disposent ni d'eau courante ni de vasque destinée à la toilette. D'un autre côté, les latrines des hauts fonctionnaires - parents, clients et, en général, individus liés à la personne du calife - ont l'eau courante, mais on ne peut en réguler le débit ; le registre décoratif se limite alors au seul bassin de marbre. Enfin, les latrines califales disposent d'eau courante dont le débit peut être contrôlé depuis celles-ci, ce qui génère le programme ornemental que nous avons décrit plus haut, incluant arc sculpté et bassin. Ces trois types d'usagers - serviteurs, hauts fonctionnaires et calife ou prince héritier - correspondent, synthétiquement parlant, à la structure politique de l'État, si bien que, à l'image d'autres éléments de la culture matérielle, le système hygiénique de Madīnat al-Zahrā' - dont la latrine peut être comprise comme la composante principale - reflète également la configuration du pouvoir dans la société califale ${ }^{35}$.

Texte traduit de l'espagnol par Patrice Cressier

35. Cette vision de l'État califal comme une structure politique extraordinairement hiérarchisée, qui est aussi celle d'E. MANZANO (Conquistadores, emires y califas. Los Omeyas y la formación de al-Andalus, Barcelone, 2006, p. 417-469), est implicite d'une façon ou d'une autre dans l'ensemble des travaux de M. Acién Almansa et s'est concrétisée de façon magistrale dans le musée de Madīnat al-Zahrā' à la conception duquel M. Acién Almansa a grandement contribué. Le haut niveau de son apport a contribué à la reconnaissance internationale de ce musée, qui a reçu les plus prestigieuses récompenses. 
Antonio Vallejo Triano - Museo de Bellas Artes, Cordoue

\begin{abstract}
Aménagements hydrauliques et ornementation architecturale des latrines de Madinat al-Zahrā' : un indicateur de hiérarchie sociale en contexte palatial
\end{abstract}

À partir du modèle omeyyade, la latrine, introduite en al-Andalus durant l'émirat, acquiert sa configuration définitive au cours du $X^{e}$ siècle, restant inchangée jusqu'à l'époque nasride. Dans ce contexte de continuité typologique, les latrines de Madinat al-Zahrā', la capitale du califat, présentent une série de progrès techniques et de singularités : adduction d'eau courante et, en conséquence, apparition d'un riche mobilier associé, destiné au contrôle du débit et à I'hygiène corporelle. La multiplicité des latrines dans la zone du qașr et leur diversité, au sein de laquelle peuvent se distinguer trois types différents, sont à l'image de l'organisation et de la hiérarchisation du palais et de la société califale en général.

Califat - décoration architecturale - hiérarchisation sociale - hygiène latrines - Madinat al-Zahrā' - Omeyyades

\title{
Hydraulic Development and Architectural Decoration in Madinat al-Zahrā's's Latrines : a Social Hierarchy Indicator in Palatial Context
}

From the Umayyad model, the latrine, introduced in al-Andalus during the emirate, acquired its definitive configuration during the tenth century, remaining unchanged until the Nasride period. In this context of typological continuity, the latrines of Madinat al-Zahrā', the capital of the caliphate, offer a series of technical progress and peculiarities: supply of running water and, later, appearance of a rich associated furniture, intended for the control of the flow and for personal hygiene. The multiplicity of latrines in the area of the qașr and their diversity, within which three different types can be distinguished, reflect the organization and the hierarchical structure of the palace and the caliphate society generally.

Architectural Decoration - Caliphate - Latrines - Madinat al-Zahrā' Personal Hygiene - Social Hierarchy - Umayyads 